\section{Avaliação das intoxicações por domissanitários em uma cidade do Nordeste do Brasil}

\author{
Poisoning with household cleaning products in a \\ city in Northeast Brazil
}

\author{
Evaluación de las intoxicaciones por productos \\ domésticos en una ciudad del Nordeste de Brasil
}

Sayonara Maria Lia Fook 1

Esthefanye Fernandes de Azevedo 1

Monalisa Maciel Costa 1

Itavielly Layany França Feitosa 1

Gerson Bragagnoli 2

Saulo Rios Mariz 2

\footnotetext{
1 Universidade Estadual da Paraíba, Campina Grande, Brasil.

2 Universidade Federal de Campina Grande, Campina Grande, Brasil.

Correspondência S. R. Mariz Universidade Federal de Campina Grande. Rua Capitão João Alves de Lira, 447, apto. 402, Campina Grande, $P B$

65400-560, Brasil. sjmariz22@hotmail.com
}

Abstract

This study analyzes toxic exposures to household cleaning products based on data from the Center for Notification and Treatment of Poisoning (CEATOX) in Campina Grande, Paraíba State, Brazil, from 2007 to 2010. The data were collected from the reporting forms from the Information System on Notifiable Diseases (SINAN), analyzed with SPSS (Version 17). Chemical identification was performed in urine samples using highresolution chromatography techniques (GC-MS and HPLC-UV). Six hundred and sixty cases of poisoning were reported, with pesticides as the principal causal agents (42.2\%). Poisoning with household cleaning products occurred mainly in children (30.1\%) and/or females (55.21\%) who were exposed to the product accidentally (55.4\%) by the oral route (82\%). These data indicate that poisoning with household cleaning products in Campina Grande should be treated with specific prevention and control measures, including evaluation of ease of access to pesticides, in order to reach the goals set by the Brazilian National Health Plan for 2012-2015.

Poisoning; Household Products; Chemical Compound Exposure

\section{Resumo}

Este trabalho analisa as exposições tóxicas a domissanitários registradas pelo Centro de Informação e Assistência Toxicológica de Campina Grande, Paraíba, Brasil, no período de 2007 a 2010. Os dados foram coletados das fichas de notificação do Sistema de Informação de Agravos de Notificação (SINAN), analisados pelo software SPSS (Versão 17), e a identificação química em amostras de urina foi feita por técnicas cromatográficas de alta resolução (CG-MS e HPLC$U V)$. Foram registradas 660 intoxicações e, considerando as variáveis de estudo, observados como principais agentes causais os agrotóxicos (42,2\%).Os intoxicados por saneantes domésticos na região são predominantemente crianças (30,1\%), elou do gênero feminino (55,21\%), que se expõem ao produto acidentalmente $(55,4 \%)$ pela via oral (82\%). Diante desses dados, conclui-se que a intoxicação por domissanitários em Campina Grande e região deve ser tratada com ações específicas de prevenção e controle junto à população, como a avaliação da facilidade de acesso aos venenos agrícolas, para que se consiga atingir as metas estabelecidas pelo Plano Nacional de Saúde, para o quadriênio 2012-2015.

Envenenamento; Produtos Domésticos; Exposição a Produtos Químicos 
Os domissanitários são substâncias ou preparações destinadas à higienização ou desinfecção de ambientes coletivos ou públicos. Na composição de saneantes domésticos pode haver a presença de substâncias cáusticas, como, por exemplo em detergentes (cloreto de benzalcônio), antissépticos (fenol), desinfetantes (ácido oxálico), desodorizantes (ácido fórmico) e alvejantes (hipoclorito de sódio). Os domissanitários podem também ser representados pelos repelentes, produtos para jardinagem amadora, agrotóxicos de uso doméstico, produtos biológicos e plantas ornamentais 1,2 .

As exposições tóxicas a esses produtos são relativamente comuns para mulheres adultas, adolescentes e, sobretudo crianças, visto que, no Brasil, os acidentes tóxicos, especialmente os não intencionais, constituem a principal causa de atendimento de emergência pediátrica.

Os dados sobre acidentes tóxicos são disponibilizados nas publicações anuais do Sistema Nacional de Informações Tóxico-Farmacológicas (SINITOX), que concentra informações registradas por 38 Centros de Informação Toxicológica do Brasil. Esse serviço é vinculado à Fundação Oswaldo Cruz (Fiocruz), e tem como atribuição primeira coordenar o processo de coleta, compilação, análise e divulgação dos casos de exposição tóxica registrados no país pela Rede Nacional de Centros de Informação e Assistência Toxicológica (RENACIAT). Os Centros filiados a essa rede estão localizados em 19 estados brasileiros 3 .

Em 2010, a RENACIAT registrou 86.700 casos de intoxicação humana e os domissanitários responderam por $12,47 \%$ dos casos $(n=10.815)$, representando o terceiro grupo mais registrado com relação às notificações ${ }^{4}$. O SINITOX informa ainda que os três principais agentes causadores de intoxicações em seres humanos no Brasil desde 1986 são medicamentos (28,3\%), animais peçonhentos/venenosos (22\%) e os domissanitários $(8,7 \%)$

No período de janeiro de 2007 a dezembro de 2010, o Centro de Informação e Assistência Toxicológica de Campina Grande (Ceatox-CG), cidade localizada no nordeste do Brasil, registrou intoxicações por alimentos, medicamentos, drogas de abuso, plantas tóxicas, animais peçonhentos, domissanitários e agrotóxicos, totalizando 7.354 ocorrências. Desse total de casos, foram atendidas $660(8,9 \%)$ intoxicações por domissanitários.

As notificações por domissanitários ocupam o terceiro lugar entre os agentes tóxicos responsáveis pelos casos atendidos no Ceatox-CG, no período analisado; assim, a relevância desse grupo de toxicante está de acordo com os dados do SINITOX, Nossos dados também se correlacio- nam aos de um estudo realizado em hospitais da região metropolitana do Rio de Janeiro, no qual os saneantes domésticos foram responsáveis por 19\% dos casos de intoxicação humana, ocupando também a terceira posição 5 .

Tais dados nos alertam para possíveis problemas no que se refere à utilização e disponibilização dos domissanitários na região em estudo, pois, no período avaliado, as exposições tóxicas por esses agentes foram inferiores apenas às ocorrências por animais peçonhentos/venenosos $(56,7 \%)$ e medicamentos (18,5\%). Essas considerações se tornam relevantes e necessitam de maior atenção das autoridades responsáveis pela fiscalização e comercialização dos saneantes domésticos.

Percebe-se, inicialmente, que os acidentes por tais produtos afetam, em sua maioria, pessoas do gênero feminino, com $55,21 \%$ dos casos ( $n=365$ ), conforme demonstra a Tabela 1. Esse dado se encontra em conformidade com as notificações da Rede Nacional de Centros do Brasil, referente ao ano de 2010 4. Tal fato é compreensível, tendo em vista que as mulheres, apesar de nas últimas décadas estarem progressivamente mais envolvidas com atividades profissionais fora da residência, ainda são as principais responsáveis pelo gerenciamento de atividades de limpeza doméstica, o que resulta num maior contato direto com esses produtos.

$\mathrm{Na}$ análise das vias de exposição, constatase o predomínio da via oral, em $82 \%(n=542)$ das intoxicações. Tal resultado é semelhante ao de um estudo realizado no Chile, no qual foram relatados cerca de 130 mil casos de intoxicação humana entre os anos de 1992 e 2002, e encontrou-se que $78,8 \%$ dos intoxicados se expuseram ao agente por via oral 6 .

A constatação de que a maioria dos casos consiste em acidentes infantis (Tabela 1) e por via oral, ressalta que medidas de prevenção de exposições tóxicas em crianças são necessárias e eficazes, como por exemplo, a utilização da embalagem especial de proteção à criança que, implantada já há algum tempo nos Estados Unidos e no Canadá, mostrou sua eficácia, uma vez que os índices de intoxicação foram reduzidos em até 35\% no período de 1969 a 1972 7,8,9,10.

Ao analisarmos a significância estatística $(\mathrm{p}<$ $0,05)$ dessa variável, podemos afirmar que o aumento da idade diminui o risco de intoxicação por esses agentes. Esse dado não causa espanto, uma vez que crianças estão mais tempo no ambiente doméstico que adultos, e também porque essa categoria engloba os menores de quatro anos, os quais estão na "fase oral" da vida, momento em que o ser humano tende a levar objetos à boca (Tabela 1). 
Caracterização dos casos de exposições tóxicas por domissanitários atendidos e notificados no Centro de Informação e Assistência Toxicológica de Campina Grande, Paraíba, Brasil, segundo as variáveis demográficas e socioeconômicas, entre os anos de 2007-2010 ( $n=660)$.

\begin{tabular}{|c|c|c|c|c|c|}
\hline \multirow[t]{3}{*}{ Variáveis demográficas e socioeconômicas } & \multicolumn{4}{|c|}{ Gênero } & \multirow[t]{3}{*}{ Valor de $p$} \\
\hline & \multicolumn{2}{|c|}{ Masculino } & \multicolumn{2}{|c|}{ Feminino } & \\
\hline & $\mathbf{n}$ & $\%$ & $\mathrm{n}$ & $\%$ & \\
\hline \multicolumn{6}{|l|}{ Faixa etária (anos) [n = 653] } \\
\hline $0-9$ & 99 & 15,2 & 97 & 14,9 & \\
\hline $10-19$ & 33 & 5,1 & 76 & 11,6 & 0,044 \\
\hline $20-29$ & 73 & 11,2 & 81 & 12,4 & \\
\hline $30-39$ & 38 & 5,8 & 50 & 7,7 & \\
\hline $40-49$ & 25 & 3,8 & 31 & 4,7 & \\
\hline $50-59$ & 13 & 2,0 & 17 & 2,6 & \\
\hline 60 anos ou mais & 10 & 1,5 & 10 & 1,5 & \\
\hline Total & 291 & 44,6 & 362 & 55,4 & \\
\hline \multicolumn{6}{|l|}{ Grau de instrução [n = 374] } \\
\hline Analfabeto & 21 & 5,6 & 22 & 5,8 & \\
\hline 1ㅇ Grau & 94 & 25,1 & 121 & 32,3 & 0,017 \\
\hline 2o Grau completo & 34 & 9,1 & 82 & 21,9 & \\
\hline Total & 149 & 39,8 & 225 & 60,2 & \\
\hline \multicolumn{6}{|l|}{ Circunstância [n = 657] } \\
\hline Acidentais & 166 & 25,3 & 200 & 30,4 & 0,665 \\
\hline Tentativa de suicídio & 120 & 18,2 & 155 & 23,6 & \\
\hline Outras & 6 & 0,9 & 10 & 1,6 & \\
\hline Total & 292 & 44,4 & 365 & 55,6 & \\
\hline
\end{tabular}

Com relação aos venenos agrícolas, houve a predominância do "chumbinho", um agrotóxico de uso agrícola, comercializado no país sob a forma exclusiva do produto Temik 150 (Aldicarbe), da empresa Bayer Cropsciences. O ingrediente ativo Aldicarbe pertence ao grupo químico Metilcarbamato de Oxima. O produto é autorizado para uso exclusivamente agrícola como inseticida, acaricida e nematicida, nas culturas de algodão, batata, café, cana-de-açúcar, citros e feijão. Acredita-se que a reavaliação de sua disponibilidade se faz necessária, dentro de um contexto de dificuldade de controle que resulta em uso irregular e indiscriminado no país como raticida, agente abortivo e ainda em tentativas de homicídio e de suicídio, acarretando um grave problema de saúde pública, de amplitude nacional, dada a facilidade de acesso, no comércio popular, a produtos contendo esse ingrediente, particularmente nos centros urbanos 11 .

Em relação à evolução dos casos, apenas 8 pacientes foram a óbito; o agente responsável foi o supracitado chumbinho, representando uma letalidade de $1,2 \%$. Os grupos químicos identificados, nas amostras de chumbinho, foram Temik
(Aldicarbe) e o Carbofuram. Ressalte-se que tal identificação, realizada em duas etapas, primeiramente mediante o uso da técnica de cromatografia líquida de alta eficiência com detector de ultravioleta (HPLC-UV) e, posteriormente, procedendo-se à confirmação dos resultados por cromatografia a gás acoplada à espectrometria de massas, resultou na confirmação inequívoca da substância química envolvida na ocorrência letal.

De posse dos dados apresentados anteriormente, foi realizada uma avaliação do risco, que é um processo sistemático pelo qual o risco, o perigo e a exposição são identificados e quantificados 12, e observou-se que os agentes causais são potencialmente perigosos, pois, apesar da baixa mortalidade encontrada, existe um risco real de exposição pela população em geral e uma significativa morbidade. O Plano Nacional de Saúde (PNS) estabelece como uma de suas diretrizes para o quadriênio 2012-2015, a redução dos riscos e agravos à saúde da população, por meio das ações de promoção e vigilância em saúde 13 . No tocante às intoxicações, propõe-se que medidas estruturantes sejam mantidas e reforçadas, 
com foco na melhoria dos indicadores, tendo em conta o grande impacto epidemiológico e assistencial de tais agravos. Entre as prioridades, estão o aperfeiçoamento do sistema público de prevenção, diagnóstico, notificação, investigação epidemiológica, atendimento e tratamento das intoxicações de populações expostas a substâncias químicas. Por fim, dentre as várias metas estabelecidas, destaca-se a redução em $50 \%$ da subnotificação de intoxicações por agrotóxicos até 2015 , sendo $10 \%$ a cada ano.

Espera-se que esse perfil oriente o planejamento e a implantação de ações preventivas de intoxicações por domissanitários, orientando e/ ou monitorando a população, para o armazenamento e a utilização adequada dos saneantes domésticos. É válido ressaltar a importância da adoção de medidas de prevenção, não somente ativas como também passivas, entre as quais destacamos a adoção de embalagens especiais de proteção à criança. Com base no conhecimento de dados epidemiológicos como os gerados por essa pesquisa, as diretrizes e metas estabelecidas pelo PNS, para o quadriênio 2012-2015, poderão ser efetivamente alcançadas.

\section{Resumen}

Este trabajo analiza las exposiciones tóxicas a produc tos domésticos registradas por el Centro de Información y Asistencia Toxicológica de Campina Grande, Paraíba, Brasil, durante el periodo de 2007 hasta 2010. Los datos fueron recogidos mediante la información proporcionada por el Sistema de Notificación de Enfermedades (SINAN) y analizados por el software SPSS (versión 17). La identificación química en muestras de orina fue realizada mediante alta resolución con técnicas de cromatografía (GC-MS y HPLC-UV). Se registraron 660 intoxicaciones $y$, teniendo en consideración las variables del estudio, se observó que los principales agentes causales eran los pesticidas $(42,2 \%)$ y que los envenenados por productos domésticos de limpieza en la región eran en su mayoría niños $(30,1 \%)$ y mujeres $(55,21 \%)$, expuesto a un producto accidentalmente $(55,4 \%)$ y por vía oral (82\%). Con estos datos, se concluye que la intoxicación por productos de uso doméstico en la región debe ser tratada con acciones específicas para la prevención y el control, como la evaluación de la facilidad del acceso a venenos agrícolas para alcanzar los objetivos establecidos por el Plan Nacional de Salud.

Envenenamiento; Productos Domésticos; Exposición a Compuestos Químicos

\section{Colaboradores}

S. M. L. Fook participou da concepção da ideia da pesquisa, redação do artigo e aprovação final do texto a ser publicado. E. F. Azevedo, M. M. Costa e I. L. F Feitosa participaram da aquisição dos dados, redação do artigo e aprovação final do texto a ser publicado. G. Bragagnoli e S. R. Mariz participaram da análise e interpretação dos dados, revisão crítica do conteúdo intelectual do artigo e aprovação final do texto a ser publicado.

\section{Agradecimentos}

Os autores agradecem à Agência Nacional de Vigilância Sanitária e ao Instituto Nacional de Criminalística pela colaboração na identificação química das amostras biológicas. 


\section{Referências}

1. Schvartsman S. Domissanitários e plantas ornamentais. In: Oga S, organizador. Fundamentos de toxicologia. 3a Ed. São Paulo: Editora Atheneu; 2008. p. 165-73.

2. Rao RB, Fulton JA. Caustic. In: Flomenbaum NE, Goldfrank LR, Hoffman RS, Howland MA, Lewin NA, Nelson LS, editors. Goldfranks: toxicologie emergencie. 8th Ed. New York: McGraw-Hill; 2006. p. 1405-16.

3. Presgrave RF, Camacho LAB, Boas MHSV. Análise dos dados dos centros de controle de intoxicação do Rio de Janeiro, Brasil, como subsídio às ações de saúde pública. Cad Saúde Pública 2009; 25:401-8.

4. Sistema Nacional de Informações Tóxico-Farmacológicas, Instituto de Comunicação e Informação Científica e Tecnológica em Saúde, Fundação Oswaldo Cruz. Estatística anual de casos de intoxicação e envenenamento. Brasil, 2010. http://www. fiocruz.br/sinitox_novo/cgi/cgilua.exe/sys/start. htm?sid=379 (acessado em 08/Out/2012).

5. Werneck GL, Hasselmann MH. Intoxicações exógenas em hospitais da região metropolitana do Rio de Janeiro. Cad Saúde Colet (Rio J.) 2005; 13: 767-78.

6. Mena C, Bettini M, Cerda P, Concha F, Paris E. Epidemiología de las intoxicaciones en Chile: una década de registros. Rev Méd Chile 2004; 132:493-9.

7. Ferreira MG, Gikas RMC, Graffs S, Scivoletto S. Abordagem epidemiológica das intoxicações alcoólicas em crianças e jovens em diversas circunstâncias, notificadas ao Centro de Controle de Intoxicações de São Paulo, no período de janeiro de 1993 a dezembro de 1997. Rev Psiquiatr Clín (São Paulo) 2000; 7:71-5.
8. Bochner R. Papel da vigilância sanitária na prevenção de intoxicações na infância. REVISA Revista Brasileira de Vigilância Sanitária 2005; 1:50-7.

9. Ramos CLJ, Targa MBM, Stein AT. Perfil das intoxicações na infância atendidas pelo Centro de Informação Toxicológica do Rio Grande do Sul (CIT/ RS), Brasil. Cad Saúde Pública 2005; 21:1134-41.

10. Werneck GL, Hasselmann MH. Intoxicações exógenas em crianças menores de seis anos atendidas em hospitais da região metropolitana do Rio de Janeiro. Rev Assoc Méd Bras (1992) 2009; 55:302-7.

11. Gerência Geral de Toxicologia, Gerência de Análises Toxicológicas, Agência Nacional de Vigilância Sanitária. Nota técnica da reavaliação do ingrediente ativo Aldicarbe. http:// portal.anvisa.gov.br/ wps/content/Anvisa+Portal/Anvisa/Inicio/Agro toxicos+e+Toxicologia/Assuntos+de+Interesse/ Monografias+de+Agrotoxicos/Monografias (acessado em Abr/2012).

12. Camargo JLV, Barros SBM. Informações científicas e avaliação do risco toxicológico. Rev Bras Toxicol 2011; 24:1-9.

13. Secretaria Executiva, Ministério da Saúde. Plano Nacional de Saúde - PNS: 2012-2015. Brasília: Ministério da Saúde; 2011. (Série B. Textos Básicos de Saúde).

Recebido em 21/Out/2012

Versão final reapresentada em 31/Jan/2013

Aprovado em 18/Fev/2013 